\title{
Why do Complications Accumulate in Individual Patients?
}

\author{
AMNON SONNENBERG* \\ The Department of Veterans Affairs Medical Center and The University of New Mexico, Albuquerque, NM, USA
}

(Received 10 December 2001; Revised 16 August 2002; In final form 20 August 2002)

\begin{abstract}
It appears as if the failure of one organ system precipitates the subsequent failure of other organ systems. The aim of the present analysis is to model such system behavior and understand why medical complications accumulate in individual patients. The human body is first modeled as being comprised of multiple subsystems, with the health of each subsystem dependent on input regarding its own health status and that of all other subsystems. In a second step, the discrete model is generalized into a continuous model that captures system failure, as well as system repair, by a first order differential equation. Failure is approximated by a logistic decline and repair is approximated by a logistic rise in health. A small drop in health of a single subsystem spreads throughout the entire system and affects its overall health. Unless counteracted by measures of therapy or repair, any time-related loss in health of individual subsystems leads to a decline in health of the entire system. The delay in onset of therapy represents the most crucial factor to influence the overall cumulative decline in health. The model suggests that medical management needs to be expeditious to minimize the cumulative time-dependent toll of illness on the entire body.
\end{abstract}

Keywords: Adverse events; Aging; Medical decision analysis; Logistic equation; Mathematical simulation; Modeling

\section{INTRODUCTION}

It appears to represent a frequently recurring clinical scenario: a patient is subjected to one minor elective procedure, a complication ensues, which subsequently leads to a second or even a third complication, and before long a relatively healthy person may turn into a severely sick patient. For instance, a patient undergoes a colonoscopy for screening of colorectal cancer, an incidental colon polyp is found and excised during the colonoscopy. (Polyps are excised because over prolonged time periods about $10 \%$ can degenerate into cancer.) The polypectomy results in a perforation of the large bowel, the patient survives the surgical closure of the colon and the treatment of the perforation-induced peritonitis, but subsequently requires assisted ventilation on the surgical intensive care unit and dies eventually from pneumonia. In a second example, a dehydrated patient is given intravenous fluids, a cellulites and phlebitis develop at the infusion site, which are cured by antibiotics. The antibiotic therapy, however, leads to pseudomembranous colitis, which represents a relatively common side-effect of antibiotic therapy. In this particular patient, the colitis further leads to massive diarrhea, loss of potassium (hypokalemia) with frequent bowel movements, and eventually atrial fibrillation (= irregular heartbeat) secondary to the hypokalemia. Such chains of interrelated events are not necessarily restricted to purely medical complications, but can also involve a multitude of other problems that are associated with general issues of patient care and hospitalization (Nolan, 2000; Reason, 2000; Weingart et al., 2000). Medications or patients are mixed up, because of poor scheduling patients become exposed to too many physically demanding procedures within a short time, without proper supervision, patients fall out of bed or incur other injuries. Frequent scheduling of investigations is sometimes driven by the medical need to expedite the clinical work-up of a very sick patient, which in itself may then contribute to a vicious cycle of a higher likelihood of subsequent system failure or death. Such potential risks associated with medical interventions abound in clinical practice. The hesitancy to admit elderly patients to the hospital or the tendency to discharge them early, reflect not only cost-effective medical strategies, but

*Address: Gastroenterology Section, Department of Veterans Affairs Medical Center 111F, 1501 San Pedro Drive SE, Albuquerque, NM 87108, USA. Tel.: + 1-505-265-1711. Ext.: 4513. Fax: + 1-505-256-5751. E-mail: sonnbrg@unm.edu 
also deep rooted concerns about the cumulative risk of complications associated with hospitalization and any type of medical management. Why do complications accumulate in individual patients? It appears as if the failure of one organ system precipitates the subsequent failure of other organ systems. The aim of the present analysis is to model such system behavior in order to understand how such patterns become possible and what mechanisms precipitate their occurrence. The first analysis comprises a discrete event modeling that uses an electronic spreadsheet to simulate health decline associated with system failure. This initial simulation is then expanded into a more general model using a differential equation that explores in addition to system decline also system repair.

\section{DISCRETE SIMULATION}

The human body is modeled as a single system comprised of multiple subsystems. Such subsystems include, for instance, heart, vasculature, lungs, kidneys, gastrointestinal tract, immune system, etc. The circles of Fig. 1 symbolize four exemplary subsystems, while the arrows symbolize influences among them (the number of four subsystems is chosen arbitrarily, and the present model does not depend on having any specific number of subsystems). Each subsystem exerts an influence on all three other subsystems, as well as on itself. A system is described in terms of its health, the value of health varying

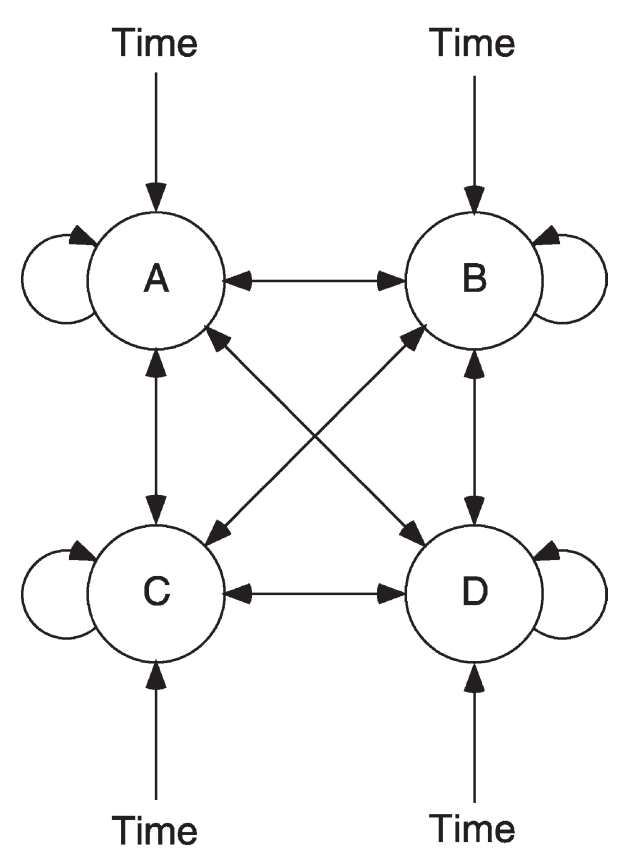

FIGURE 1 Model of a human body system comprised of four subsystems (A) through (D). The arrows indicate inhibitory influences among the four separate subsystems. The health of each subsystem depends on input regarding its own status, as well as all three other subsystems. In addition, each subsystem may be influenced by the passage time. between 0 and 1 . Health is defined as the ability of a system or subsystem to carry out its function. The overall health of the entire system represents the weighted sum of the health values of its subsystems:

$$
H=\sum_{\mathrm{i}} w_{\mathrm{i}} H_{\mathrm{i}}
$$

where $w_{\mathrm{i}}$ and the $H_{\mathrm{i}}$ represent the weights and the health values, respectively, associated with each individual subsystem. The sum of weights is $100 \%$. Here, capital subscripts are used to denote different subsystems, and superscripts are used to denote consecutive time periods. The time frame of the analysis is divided into equal increments of time units, during which the health of the system changes. Between the time points $t^{1}$ and $t^{2}$ the health of the subsystem A equals the initial health minus the fraction that has failed:

$$
H_{\mathrm{A}}^{2}=H_{\mathrm{A}}^{1}-H_{\mathrm{A}}^{1} \mathrm{FR}^{1} .
$$

$H_{\mathrm{A}}^{1}$ and $H_{\mathrm{A}}^{2}$ represent the health of the subsystem $\mathrm{A}$ at the beginning and at the end of the time interval, respectively, and $\mathrm{FR}^{1}$ represents the failure rate at the beginning of the time interval. The failure rate per time interval varies between 0 and $100 \%$. The model is built to capture the fact that healthy subsystems protect each other from failure, whereas failed or less healthy subsystems expose all other subsystems also to an increased failure risk. On one hand, if all subsystems are healthy and function well, the failure rate should equal 0 . On the other hand, if all subsystems fail, the overall failure rate should equal $100 \%$. This behavior is captured by the following formula:

$$
\mathrm{FR}^{1}=1-\sum_{\mathrm{i}} f_{\mathrm{i}} H_{\mathrm{i}}^{1},
$$

and

$$
100 \%=\sum_{\mathrm{i}} f_{\mathrm{i}}
$$

where $f_{\mathrm{i}}$ represent the contribution of health associated with individual subsystems to the overall failure rate FR. As the health values of the individual subsystems change with each cycle so does the failure rate. Once Eq. (2) has been used to re-calculate the new health values $H_{\mathrm{i}}^{2}$ of all $\mathrm{i}$ subsystems, a new failure $\mathrm{FR}^{2}$ is calculated, which in turn is then utilized to derive $H_{\mathrm{i}}^{3}$. The long-term behavior of the system is calculated by a repetitive iteration of Eqs. (2) and (3). If one wants to account also for the natural deterioration of any given system with the passage of time and include a time-dependent loss in health, Eq. (4) changes to:

$$
100 \%=f_{t}+\sum_{\mathrm{i}} f_{\mathrm{i}}
$$

This adjustment guarantees that, even with perfect health of all subsystems, the failure rate associated with each cycle is greater than 0 , and the overall system exhibits an age-related decline over time. 


\section{RESULTS OF DISCRETE SIMULATION}

The model shown in Fig. 1 and outlined by Eqs. (1) through (5) can be easily executed on an electronic spreadsheet, such as Excel $^{\circledR}$ from Microsoft (Redmond, WA). Different columns of the spreadsheet represent different subsystems, and consecutive rows represent consecutive time cycles. Equations (2) and (3) serve to calculate the contents of each cell using the contents of cells from the preceding row, that is, a previous time cycle. Out of convenience (and without any loss of generality) identical weights were used for all subsystems.

Figure 2 shows the system behavior under various conditions. If all subsystems start with perfect health $H_{\mathrm{i}}=1$ and $f_{t}=0$, the overall system stays healthy for ever. This is indicated by the straight line in the left panel. The introduction of a time-related loss in health $\left(f_{t}>0\right)$ changes the time-dependent health of the system from a straight line to a curve shaped like the inverted letter $\mathrm{S}$. The initiation of decline depends the magnitude of $f_{t}$. Larger values of $f_{t}$ shift the decline to the left towards earlier time periods, but leave its shape largely unchanged. This temporal pattern can be explained as follows. At first, the time-dependent loss in health does not amount to much, and it does not affect the overall system to any large extent. Because the failures accumulate only very slowly, they are hardly noticeable during the early time periods. However, once the health of the individual subsystems has dropped by more than $1 \%$, Eq. (3) results in an ever increasing failure rate and the overall system health starts to drop precipitously. The failure rate loses steam only after most of the health associated with each of the subsystems has been wasted, and the steep decline in system health peters out towards the end.
The introduction of partial loss of health in any of the system's four subsystems is shown in the right panel of Fig. 2. It has a similar influence on the system's overall behavior as the increase in the time-dependent failure secondary to the time constant $f_{t}$, and its mode of action is similar as outlined above. By reducing the health of all other subsystems as well, a small loss in health limited initially to a single subsystem accumulates within the entire system. An increase in the number of subsystems increases the overall stability of the system, and it takes longer for any loss in health affecting one or few subsystems to cause the demise of the entire system. However, the general characteristics of the system and the shape of its time-dependent decline in health remain unaffected by the number of subsystems.

\section{CONTINUOUS ANALYSIS}

In the previous analysis, system health was simulated on a spreadsheet as a set of recursive difference equations. The following section serves to show the relationship between the logistic function and the system behavior outlined above. In case of a single system, Eqs. (2) and (3) can be stated as

$$
H^{2}-H^{1}=H^{1}\left(1-f H^{1}\right),
$$

or as the following differential equation:

$$
\frac{\partial H}{\partial t}=-c_{1} H(1-f H)
$$

where $H$ represents health, $f$ the failure rate, and $c_{1}$ some arbitrary time constant. This equation would also represent system health, if all subsystems were
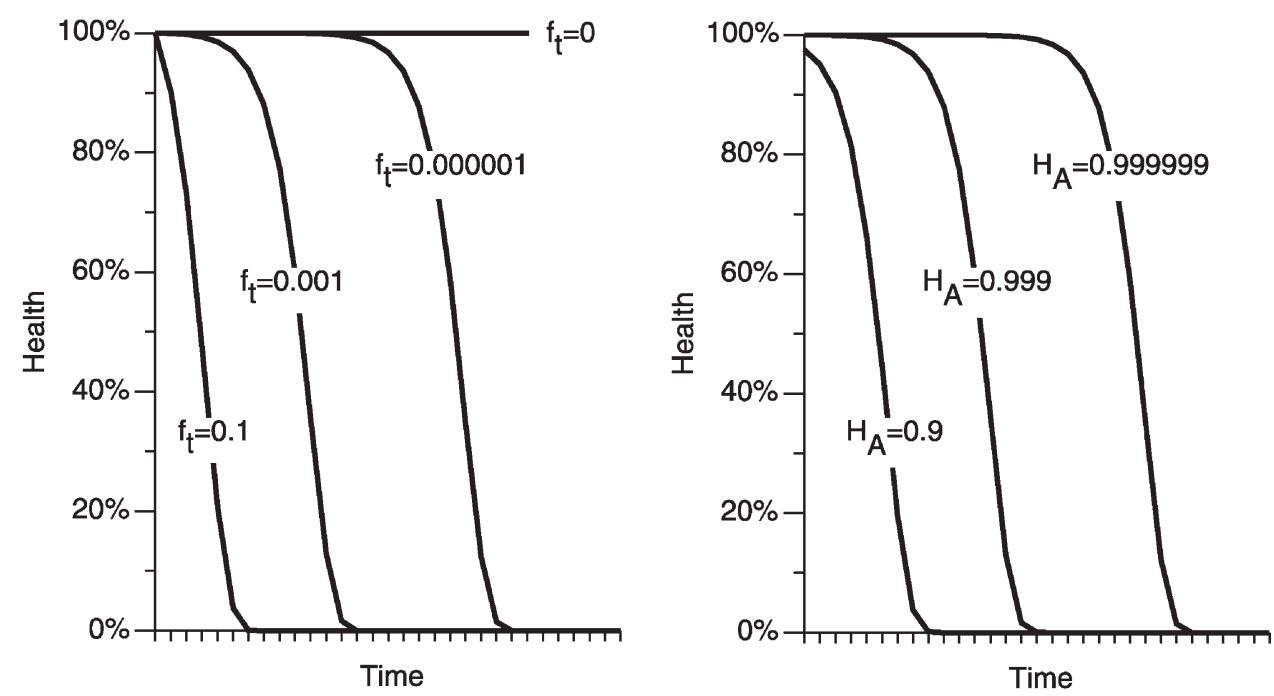

FIGURE 2 System health as function of time. In the left panel, the time constant $f_{\mathrm{t}}$ is varied between $f_{\mathrm{t}}=0$ and $f_{\mathrm{t}}=0.1$. In the right panel, the initial loss in health associated with one of the four subsystems is varied between $H_{\mathrm{A}}=0.9$ and $H_{\mathrm{A}}=0.999999$. 
characterized by an identical failure rate and contributed equally to the overall system health. Its integration yields (Beyer, 1981):

$$
H=\frac{c_{2} e^{-c_{1} t}}{1+f c_{2} e^{-c_{1} t}} .
$$

The second constant $c_{2}$ is given by a boundary condition, such as $H\left(t_{0}\right)=H_{0}$. This time dependent logistic function of system health is characterized by a similar curve as shown in Fig. 2.

In most instances, the disease process does not result in a complete loss of health, but may stop at some level $(a)$ located between 0 and 1 . For instance, the injury to a limb, even if it leads to amputation, would not bring down the entire system. Rather than modeling the decline in health proportional to $H$, the decline is assumed to be proportional to its distance from the level $(H-a)$. Equation (6) then changes to

$$
\frac{\partial H}{\partial t}=-c_{1}(H-a)(1-f H)
$$

and its solution changes to

$$
H=\frac{a+c_{2} e^{-(1-f a) c_{1} t}}{1+f c_{2} e^{-(1-f a) c_{1} t}} .
$$

The onset of a decline in health is usually met by counter-measures to restore the initial healthy state. The counter-measures could relate to physiologic mechanisms aimed at repair or fending off disease, such as an immune response or wound healing. They could also be initiated from the outside through medical therapy. The changes in health following repair and therapy can again be modeled as a logistic progress. In contrast with disease and the negative influence of time-dependent changes, therapyinduced changes in health are positively correlated with time. Therapy could be associated with a complete restoration of health or stop at a level $(b)$ beyond which further improvement is not achievable. The corresponding differential equation is

$$
\frac{\partial H}{\partial t}=c_{3} H(b-H)
$$

and its solution is

$$
H=\frac{b}{1+c_{4} e^{-b c_{3} t}} .
$$

Again, the time constant $c_{3}$ and the constant $c_{4}$ represent characteristics of the therapeutic process given by its boundary conditions. This time dependent logistic function of health improvement under therapy is shown in Fig. 3. At any given point in time, the actual health status would represent the sum of two counteracting mechanisms, that is, a disease-related decline and a therapy-induced rise. In general, therapy and repair mechanisms are initiated with some time delay $T$ after

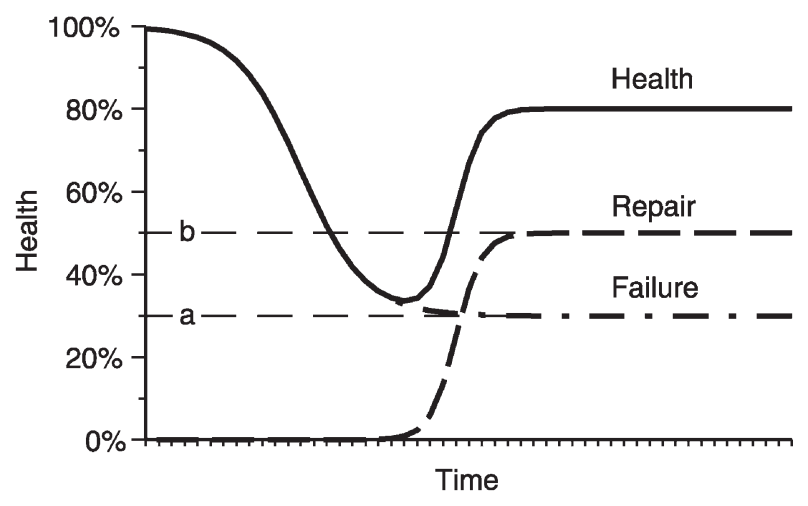

FIGURE 3 System health modeled as the joint influence of failure (disease) and repair (therapy). Failure is calculated as a logistic decline and repair is calculated as a logistic growth.

the onset of the disease. Hence, combining Eqs. (9) and (11) and including a time delay in the second term depicting therapy yields:

$$
H=\frac{a+c_{2} e^{-(1-f a) c_{1} t}}{1+f c_{2} e^{-(1-f a) c_{1} t}}+\frac{b}{1+c_{4} e^{-b c_{3}(t-T)}} .
$$

\section{RESULTS OF CONTINUOUS ANALYSIS}

Figure 4 shows four examples of disease behavior modeled with Eq. (12). If disease behavior and repair are characterized by similar time constants and if there is little delay in initiation of repair after disease onset, health will become only marginally affected as indicated by the shallow depression depicted in panel A. In case of severe disease with progressive deterioration, any prolonged delay in the initiation of therapy will cause a more pronounced depression of health (panel B). Many chronic diseases may be characterized by a "bath tub" pattern as depicted in panel $\mathrm{C}$. The disease process results in diminished health that can remain depressed for an extensive time period until (with much delay) therapy becomes implemented. In panel $\mathrm{D}$, a rapid disease progress plus a slow and only partial disease resolution serve to show that one can use Eq. (12) to combine a large variety of different disease behavior and therapy.

The patterns depicted by the four curves of Fig. 4 are influenced by the various constants of Eq. (12). The two time constants $c_{1}$ and $c_{3}$ determine the speed of disease progression and the counteracting repair mechanism, that is, the slope of decline and the slope of rise, respectively. The time delay $T$ determines the overall length of the depression. Lastly, the constant a determines the absolute amount of fall in health, and the sum $(a+b)$ determines the level to which health can rise again after successful therapy.

In yet another subset of diseases, the amount of achievable repair $b$ may depend on the delay in the onset of therapy. With little or no delay $b=1$, that is, complete 

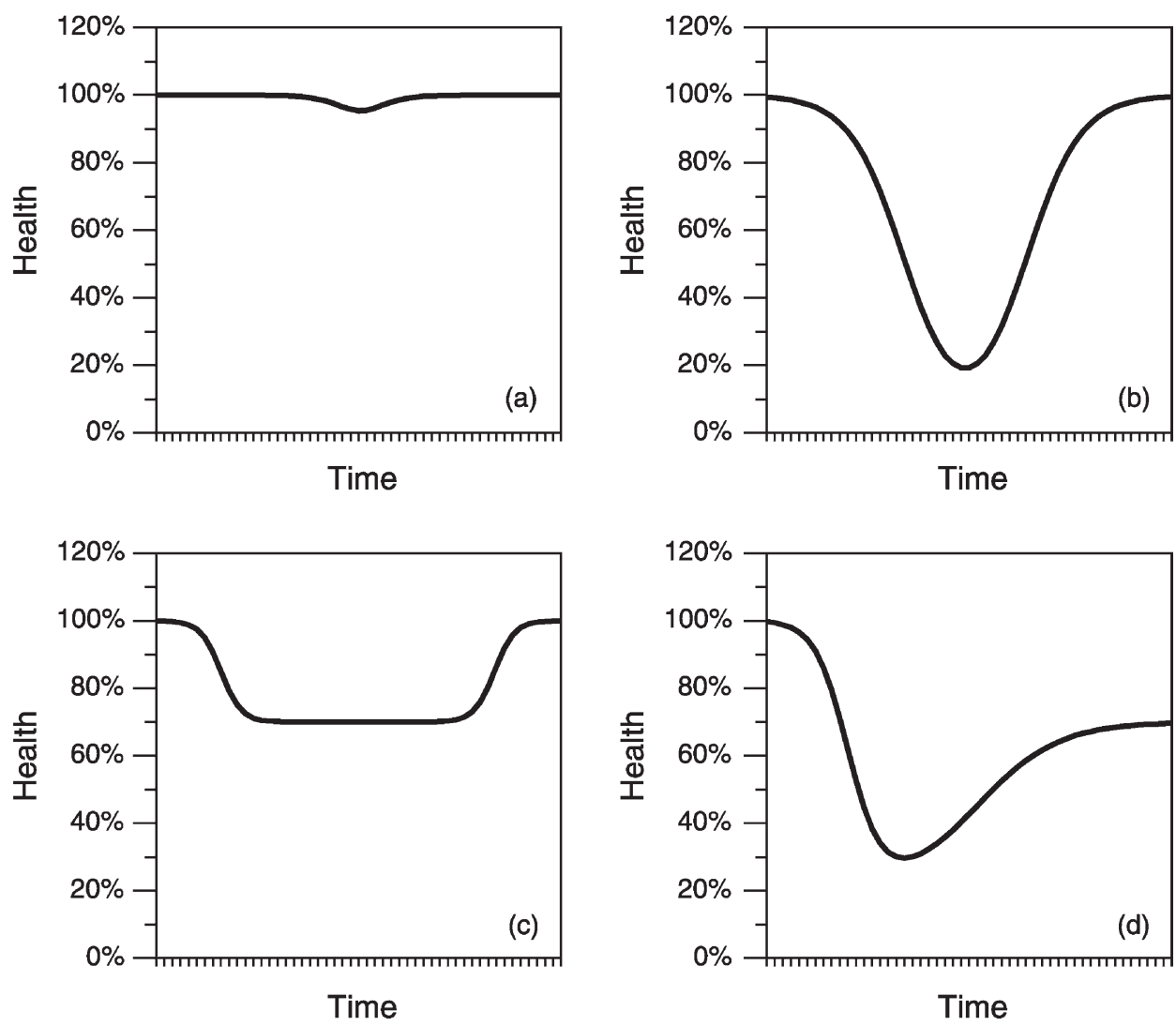

FIGURE 4 Various patterns of health behavior under the joint influence of failure and repair.

resolution is still possible, whereas long delays may lead to ineffective therapy with $b=0$. One could model such disease behavior by expressing a revised $\mathrm{b}$ as function of $T$, for instance, $b_{\text {new }}=c_{5} b / T$.

\section{DISCUSSION}

Failure of medical systems has come under increased scrutiny. The British Medical Journal devoted an entire issue to the topic and reports about medical failures have been dealt with in the lay press (Pear, 1999; Nolan, 2000; Reason, 2000; Weingart et al., 2000). Various studies from the United States, Britain and Australia have shown that $4-11 \%$ of all hospitalized patients experience adverse effects of medical intervention (Brennan et al., 1991; Leape et al., 1991; Wilson et al., 1995; Thomas et al., 2000; Vincent et al., 2001). Based on the American data, it was estimated that $69 \%$ of injuries among hospitalized patients were caused by errors (Leape et al., 1991). The Australian data suggested similarly that $51 \%$ of all adverse events associated with hospital admissions were preventable (Wilson et al., 1995). Not in all instances can the adverse event be related unequivocally to one particular procedure, and just being hospitalized or undergoing tests and medical procedures can expose patients to an appreciable number of risks. While the majority of subjects shoulder this added risk well and tolerate occasional adverse events with little or no residuals, there is a subgroup of patients in whom complications appear to accumulate and who rapidly proceed from minor to major medical disasters.

The aim of the present study was to characterize the underlying mechanisms that contribute to the accumulation of complications in individual patients. First, a mathematical model was developed to simulate how any partial loss in system function can weaken the entire system and make it more susceptible to further failure. In a second step, the discrete simulation was advanced to a continuous model that captures system failure, as well as its subsequent repair. Failure was approximated by a logistic decline, and the repair was approximated by a logistic rise of health. This model accounts for a large variety of disease patterns that can be observed in clinical practice. The underlying stochastic nature of system failure and system repair have been utilized in reliability engineering to model the time-dependent behavior of mechanical or electronic systems and their survival (Leitch, 1995). Such techniques may also provide possible avenues for further development of the present analysis.

The time-dependent behavior of the model without repair resembles similar patterns that have been found to describe aging and survival in biologic populations (Strehler, 1977; Friess, 1980). The similarity reflects 
the underlying principle of cumulative errors that lead to eventual system failure. In the natural process of aging and time-dependent decline, small errors constantly occur in a multitude of interdependent subsystems. The errors accumulate up to levels that make it increasingly more difficult for the system to cope with them and that eventually result in its death. A similar but much accelerated process underlies the accumulation of adverse medical events in susceptible patients. A patient is made more susceptible to further damage and failure by an agedependent loss in health or previous encounters with adverse events that have already reduced a patient's overall well-being.

The sequence of consecutive adverse events, which may befall individual patients does not represent just bad luck, a whim of fortune, or possibly a biased perception by the physician, but a real phenomenon with a clear-cut underlying cause. In clinical practice, many failures become averted by timely therapy and medical repair before any major loss in health spreads through the system and affects other organs. The delay in onset of therapy represents the most important factor to influence the cumulative decline in health. Prophylaxis initiated before the onset of disease or therapy begun shortly after the diagnosis of disease result in no or only minor reductions in health. Delayed therapy can lead to a prolonged state of diminished health or a even a drop in health to levels from which reversal through therapy is no longer possible.

What are the medical consequences of such insights? The present model does not lead to new hypotheses about disease or means to predict disease behavior. However, not all modeling needs to result in clinical applicability to be useful. Mathematical modeling can also be done to understand medical phenomena, which is often a sufficient goal in by itself. The present model helps to conceptualize health as an interaction between system failure and repair and aids in explaining the principles that govern health. On a more practical side, the model emphasizes the importance of speed in disease management. Medical management needs to be fast and expeditious to minimize the cumulative time-dependent toll of illness on the entire body. Moreover, medical management needs to avoid as much as possible the infliction of additional damage through diagnosis and therapy. The side-effects of medical intervention are often considered a justifiable means to reach a therapeutic or curative end. Since the system failure is a time-dependent and self-perpetuating process, however, the damage inflicted by medical intervention may soon reach levels that cannot be reversed in spite of successful therapy. Lastly, even the most focused specialists need to remain concerned about the seemingly trivial systemic consequences of their interventions. The break-up of medicine into multiple subspecialties has improved training and expertise of physicians treating rare diseases and administrating delicate procedures. In concentrating on one organ system, however, one can easily forget that mistakes and complications of a single organ eventually affect the whole body.

\section{References}

Beyer, W.H. (1981) CRC Standard Mathematical Tables, 26th Ed. (CRC Press, Boca Raton), p. 291.

Brennan, T.A., Leape, L.L., Laird, N.M., Herbert, L., Localio, A.R., Lawthers, A.G., Newhouse, J.P., Weiler, P.C. and Hiatt, H. (1991) "Incidence of adverse events and negligence in hospitalized patients: results of the Harvard medical practice study I", New England Journal of Medicine 324, 370-376.

Friess, J.F. (1980) "Aging, natural death, and the compression of morbidity", New England Journal of Medicine 303, 130-135.

Leape, L.L., Brennan, T.A., Laird, N.M., Lawthers, A.G., Localio, A.R., Barnes, B.A., Hebert, L., Newhouse, J.P., Weiler, P.C. and Hiatt, H. (1991) "Incidence of adverse events and negligence in hospitalized patients: results of the Harvard medical practice study II", New England Journal of Medicine 324, 377-384.

Leitch, R.D. (1995) Reliability Analysis for Engineers. An Introduction (Oxford University Press, New York), pp. 174-193.

Nolan, T.W. (2000) "System changes to improve patient safety", British Medical Journal 320, 771-773.

Pear, R. (1999) "Report details medical errors in V.A. hospitals", New York Times 19, 1999.

Reason, J. (2000) "Human error: models and management", British Medical Journal 320, 768-770.

Strehler, B.L. (1977) Time, cells, and aging (Academic Press, New York), pp. 103-124.

Thomas, E.J., Studdert, D.M., Burstin, H.R., Orav, E.J., Zeena, T., William, E.J., Howard, K.M., Weiler, P.C. and Brennan, T.A. (2000) "Incidence and types of adverse events and negligent care in Utah and Colorado in 1992", Medical Care 38, 261-271.

Vincent, C., Neale, G. and Woloshynowych, M. (2001) "Adverse events in British hospitals: preliminary retrospective record review", British Medical Journal 322, 517-519.

Weingart, S.N., Wilson, R.M., Gibberd, R.W. and Harrison, B. (2000) "Epidemiology of medical error", British Medical Journal 320, 774-777.

Wilson, R.M., Runciman, W.B., Gibberd, R.W., Harrison, B.T., Newby, L. and Hamilton, J.D. (1995) "The quality in Australia healthcare study", Medical Journal of Australia 163, 458-471. 


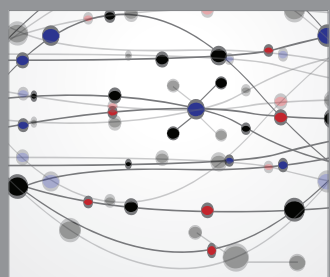

The Scientific World Journal
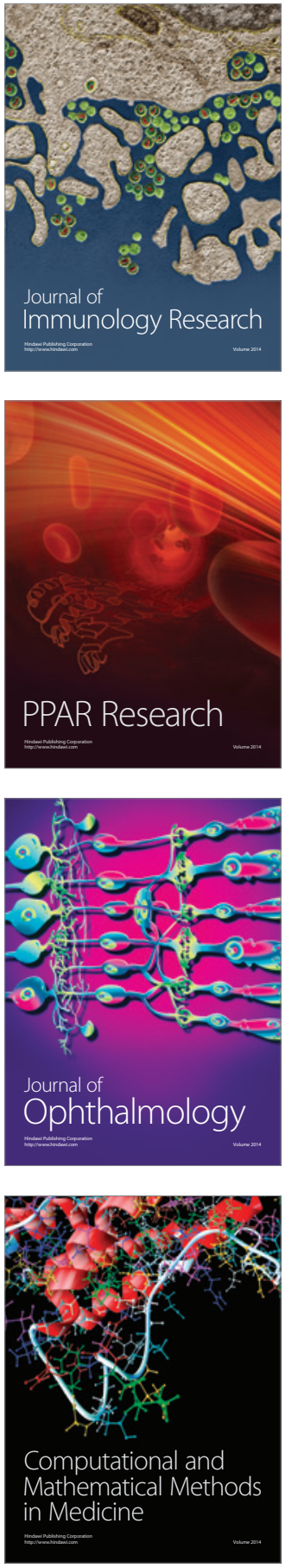

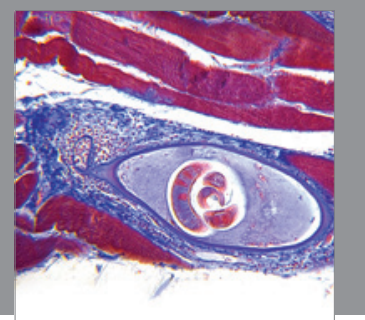

Gastroenterology

Research and Practice
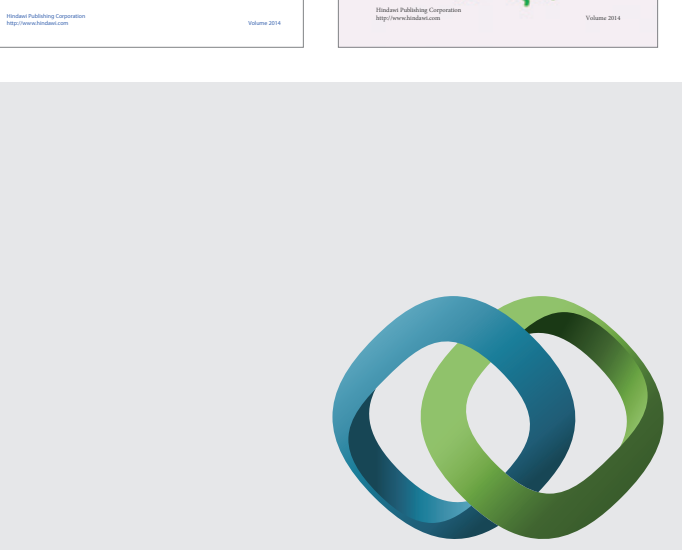

\section{Hindawi}

Submit your manuscripts at

http://www.hindawi.com
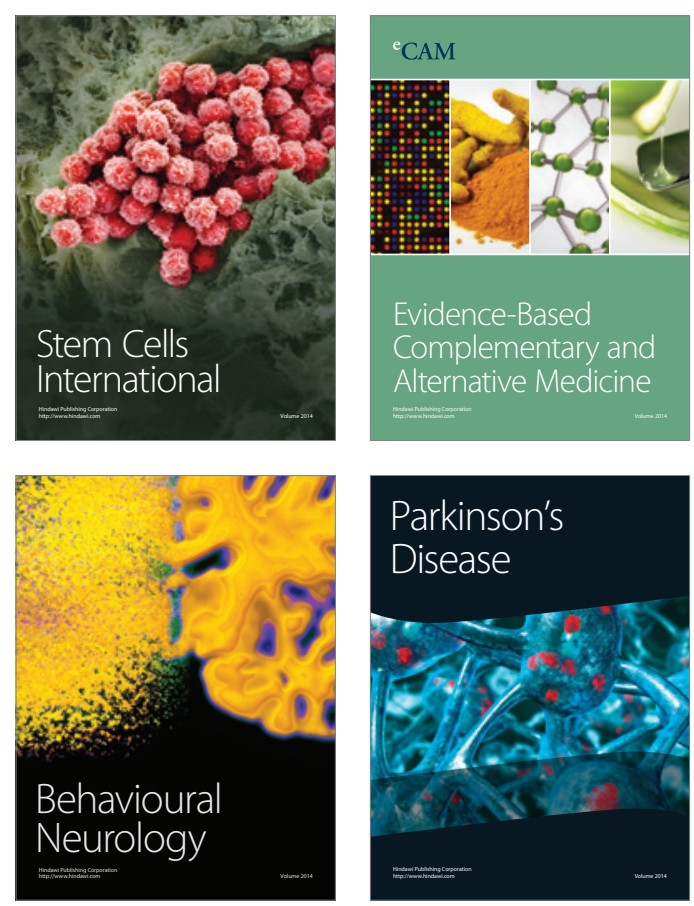

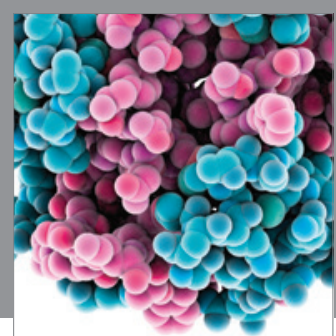

Journal of
Diabetes Research

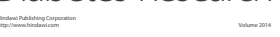

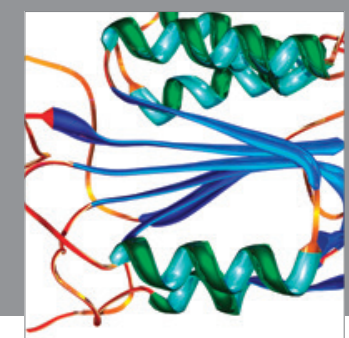

Disease Markers
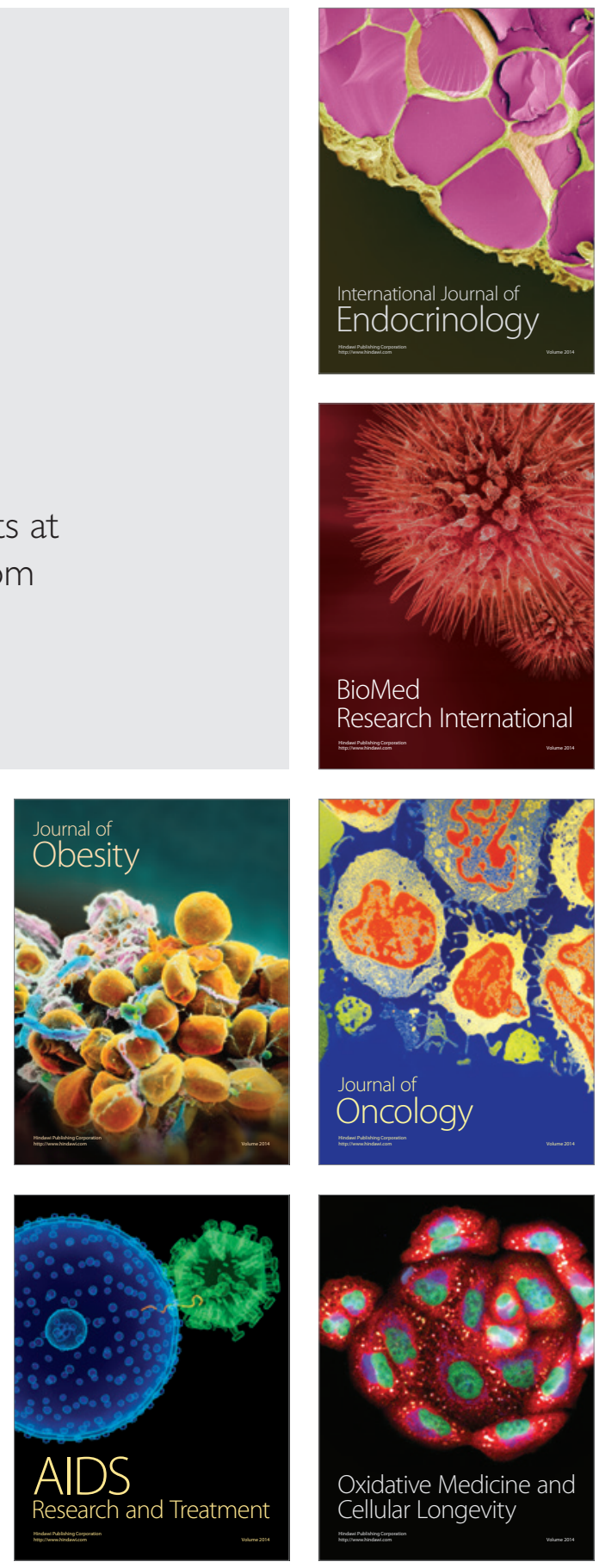